La Revue

des Droits

de l'Homme

\section{La Revue des droits de l'homme}

Revue du Centre de recherches et d'études sur les droits fondamentaux

Actualités Droits-Libertés | 2021

\title{
Les fausses notes du souverainisme juridique
}

À propos de l'arrêt de l'assemblée du Conseil d'État du 21 avril 2021

\section{Vincent Sizaire and Jean-Philippe Foegle}

\section{(2) OpenEdition}

\section{Journals}

Electronic version

URL: https://journals.openedition.org/revdh/12023

DOI: 10.4000/revdh.12023

ISSN: 2264-119X

\section{Publisher}

Centre de recherches et d'études sur les droits fondamentaux

\section{Electronic reference}

Vincent Sizaire and Jean-Philippe Foegle, "Les fausses notes du souverainisme juridique", La Revue des droits de l'homme [Online], Actualités Droits-Libertés, Online since 21 June 2021, connection on 06 December 2021. URL: http://journals.openedition.org/revdh/12023; DOI: https://doi.org/10.4000/ revdh. 12023

This text was automatically generated on 6 December 2021.

Tous droits réservés 


\title{
Les fausses notes du souverainisme juridique
}

\author{
À propos de l'arrêt de l'assemblée du Conseil d'État du 21 avril 2021
}

\author{
Vincent Sizaire and Jean-Philippe Foegle
}

1 Une fois de plus, le contraste est saisissant. Alors que l'arrêt d'assemblée du 21 avril dernier, par lequel le Conseil d'État s'est prononcé sur la conformité au droit européen des dispositions nationales organisant l'interception massive des données de connexion n'aura donné lieu en France qu'à une médiatisation limitée et complaisante, cette décision a suscité en dehors de nos frontières une salve de critiques d'une rare virulence. Qualifiée outre-Manche de «Frexit» et de «solution magique ${ }^{1}$, brocardée outre-Rhin comme la marque d'un «foyer d'infection » au sein de l'ordre juridique de l'Union européenne, elle vient une nouvelle fois alimenter la chronique acerbe de notre singulier gallicanisme juridique national ${ }^{2}$. Une telle volée de bois vert est-elle justifiée ?

2 Certes, le Conseil d'État s'est refusé à "emboiter le pas au joueur de flûte de Karlsruhe»" pour, à l'instar de la cour constitutionnelle allemande, prétendre substituer à celle de la Cour de Luxembourg sa propre interprétation des traités européens ${ }^{3}$. Mais, alors qu'il était appelé à se prononcer après de nombreux arrêts ayant statué sur la conformité de la surveillance dite «de masse» aux droits fondamentaux consacrés par le Droit de l'Union européenne, l'arrêt du 21 avril apparaît en deçà d'une suite jurisprudentielle qui avait pu, un temps, tracer l'horizon d'une meilleure articulation entre libertés numériques et techniques de renseignement.

Dans un contexte marqué par les révélations du lanceur d'alerte Edward Snowden en 2013, nombre de juridictions suprêmes européennes ont adopté des décisions visant à renforcer la protection des données personnelles des citoyen-ne-s, notamment face aux services de renseignement. Au sein de l'Union européenne, après la décision Digital Rights Ireland par laquelle la Cour de justice de l'Union européenne a invalidé la directive du 15 mars 2006 imposant la conservation généralisée des données de connexion des internautes en ce qu'elle n'était pas soumise à l'autorisation préalable d'un juge ${ }^{4}$, plusieurs juridictions nationales ont censuré des lois sur la surveillance électronique sur le fondement de la Charte des droits fondamentaux, notamment au 
Royaume-Uni ${ }^{5}$. Dans un second temps, la Cour de justice de l'Union européenne a invalidé la décision 2000/520/CE relative aux transferts de données à caractère personnel vers les États-Unis, fondant la prohibition de la surveillance de masse sur une double garantie liée au droit à la vie privée et à la protection des données personnelles ${ }^{6}$ et au droit au recours effectif. Puis, par deux arrêts des 17 décembre 2016 et 6 octobre 2020, elle a limité rigoureusement les possibilités de surveillance massive des réseaux, tout en posant de solides exigences procédurales pour assurer l'effectivité de son contrôle ${ }^{7}$. La Cour juge ainsi qu'une surveillance généralisée et indifférenciée n'est conforme au droit de l'Union que si elle tend à «la sauvegarde de la sécurité nationale » et qu'une surveillance ciblée des données de trafic et de localisation ne l'est que si elle a pour objet «la lutte contre la criminalité grave et de la prévention des menaces graves contre la sécurité publique ». En toute hypothèse, la surveillance ne peut être ordonnée que sur le fondement d'une menace effective, pour une durée limitée et être soumise au contrôle effectif d'une juridiction ou d'une entité administrative indépendante ${ }^{8}$.

Une telle jurisprudence invitait nombre d'États membres - au premier rang desquels la France - à renforcer significativement l'encadrement de l'activité de leurs services de renseignement. Le message avait d'ailleurs été parfaitement compris par ces derniers, dont la mobilisation a conduit le gouvernement à solliciter le Conseil d'État pour qu'il écarte purement et simplement l'application du droit de l'Union européenne ${ }^{9}$. C'est dans ce contexte très particulier que les juges du Palais-Royal étaient appelés à se prononcer à leur tour. Formellement saisis d'une demande d'abrogation du décret du 29 janvier 2016 relatif aux techniques de recueil de renseignements, il leur appartenait plus précisément de se prononcer sur la conformité de sa base légale, à savoir l'ensemble des dispositions organisant la surveillance des réseaux numériques ${ }^{10}$. Quand d'autres juridictions suprêmes - à l'image de la Cour constitutionnelle belge ${ }^{11}$ - ont fait leur la solution de la Cour de justice de l'Union européenne, ils ont privilégié une approche préservant une significative marge d'appréciation aux autorités nationales. C'est ainsi que les exigences européennes ne sont prises en compte que dans la mesure où elles restent compatibles avec le cadre constitutionnel français - tel qu'interprété par le juge administratif. Si cette approche conduit en l'espèce le Conseil d'État à appliquer une large partie de la jurisprudence du juge de Luxembourg, censurant en conséquence le cadre juridique national régissant la conservation des données de connexion $^{12}$ des internautes (I), elle véhicule une forme de souverainisme juridique qui fragilise la protection européenne des droits et libertés des citoyen-ne-s (II).

\section{I - Une application conditionnelle des exigences européennes}

5 Résistant à la demande pressante qui lui était faite par le gouvernement français d'écarter la jurisprudence de la Cour de Justice en ce que cette dernière aurait méconnu la répartition des compétences entre l'Union européenne et les États membres, le Conseil d'État rappelle qu'il ne lui appartient pas «de s'assurer du respect, par le droit dérivé de l'Union européenne ou par la Cour de justice elle-même, de la répartition des compétences entre l'Union européenne et les États membres $»^{13}$. En revanche, dans le prolongement de l'arrêt Arcelor de $2007^{14}$, il considère que lorsque l'invalidation d'une norme nationale contraire à une norme internationale est susceptible de priver de 
garantie une exigence constitutionnelle et qu'il "n'existe pas de règle ou de principe général du droit de l'Union européenne garantissant l'effectivité du respect de la disposition ou du principe constitutionnel invoqué ", l'application du droit interne ne peut être écartée ${ }^{15}$. $\mathrm{Au}$ bénéfice de cette interprétation, le Conseil juge ainsi que dans la mesure où les objectifs de valeur constitutionnelle de «sauvegarde des intérêts fondamentaux de la Nation ", de "recherche des auteurs d'infractions pénales" de "prévention des atteintes à l'ordre public » ne bénéficient pas en droit européen d'une protection équivalente à celle que garantit la Constitution, il lui appartient de s'assurer que lesdits objectifs ne sont pas mis en péril par les conditions posées par la Cour de justice de l'Union européenne à la captation des données de connexion par les services de renseignement ${ }^{16}$.

Si cette grille de lecture le conduit à faire siennes les exigences procédurales du juge européen (A), elle lui permet de valider une conception sensiblement plus extensive des motifs permettant de recourir à la surveillance généralisée de notre activité numérique (B).

\section{A. La prise en compte des garanties procédurales}

7 S'il prend formellement garde de préciser que "cela n'est pas susceptible de priver de garanties effectives les exigences constitutionnelles » invoquées par le gouvernement, le Conseil d'État est conduit à censurer le cadre règlementaire national en ce qu'il ne respecte pas les garanties procédurales imposées par le juge européen.

Il s'agit, en premier lieu, de l'exigence d'un contrôle effectif et contraignant par une autorité indépendante. Relevant que l'avis donné par la Commission nationale de contrôle des techniques de renseignement (CNCTR) n'est que facultatif, le Conseil est ainsi conduit à écarter l'application de la loi nationale en ce qu'elle ne donne pas un caractère impératif audit avis, que celui-ci soit requis pour l'accès en temps différé aux données de connexion par les services de renseignement, la mise en œuvre de traitements automatisés lorsqu'elle aboutit à l'identification de personnes dont les données sont susceptibles de révéler une menace à caractère terroriste ou, enfin, la mise en œuvre du recueil en temps réel des données de trafic et de localisation ${ }^{17}$. Dans cette dernière hypothèse, le juge administratif suit en outre la Cour de justice de l'Union européenne en exigeant non seulement un avis contraignant, mais encore un avis préalable.

En deuxième lieu, le Conseil d'État vient consacrer explicitement l'obligation d'information des personnes surveillées. Cette exigence, qui s'impose dès le moment où cette information n'est pas susceptible de compromettre les enquêtes menées par ces autorités, est posée par le juge de Luxembourg comme une garantie permettant aux personnes concernées de contester a posteriori le bien-fondé de cette surveillance ${ }^{18}$. Procédant à une interprétation constructive des dispositions de la loi $\mathrm{n}^{\circ}$ 78-17 du 6 janvier 1978 relative à l'informatique, aux fichiers et aux libertés, le Conseil juge ainsi qu'elles «doivent être regardées comme prévoyant, le cas échéant, l'information des personnes ayant fait l'objet d'un traitement de leurs données personnelles dans le cadre d'une des techniques de renseignement mentionnées aux articles L. 851-1 à L. 851-4 du code de la sécurité intérieure pour autant que et dès le moment où cette communication n'est pas susceptible de compromettre les missions qui incombent aux services de renseignement $»^{19}$. S'il s'agit là d'une indéniable avancée, sa portée doit toutefois être nuancée dès lors que les juridictions européennes relient étroitement l'efficacité du contrôle à la prévisibilité de la 
législation, qui doit être a minima rédigée avec suffisamment de clarté pour permettre aux individus de savoir dans "quelles circonstances et sous quelles conditions elle habilite la puissance publique à prendre pareilles mesures secrètes $»^{20}$. Or les motifs de surveillance étant en droit français particulièrement extensifs, cette exigence de prévisibilité ne saurait, tant s'en faut, être aujourd'hui regardée comme satisfaite.

En dernier lieu, conformément aux exigences du juge européen, le Conseil d'État impose au Gouvernement de procéder, sous son contrôle, à un réexamen annuel de l'existence "de la persistance d'une menace grave, réelle et actuelle ou prévisible, pour la sécurité nationale » justifiant la conservation et l'exploitation générale et indifférenciée de l'ensemble de nos données de connexion ${ }^{21}$. S'il s'agit d'un apport non négligeable à l'encadrement de l'action des services de renseignement, la question se pose des modalités concrètes du contrôle qui pourra être effectué par le juge administratif. En effet, en l'état du droit positif, c'est uniquement dans le cadre d'une saisine de la CNCTR ou d'une personne s'estimant surveillée illégalement que le Conseil d'État peut prendre directement connaissance de documents couverts par le secret de la défense nationale ${ }^{22}$. En dehors de cette hypothèse particulière, ledit secret reste opposable au juge $^{23}$, ce qui empêche celui-ci de forcer l'administration à communiquer des informations collectées par les services de renseignement. Dans l'hypothèse d'une absence de production de ces informations confidentielles, le juge administratif sera amené à statuer sur le fondement des seuls éléments en sa possession ${ }^{24}$, et se prononcer en se fondant sur des "présomptions précises et concordantes». Certes, ce cadre juridique a pu conduire à interpréter le silence de l'administration en sa défaveur lorsque celle-ci n'avait produit «aucun élément de nature à justifier que la communication serait susceptible de mettre en cause la sûreté de l'État, la défense ou la sécurité publique " ${ }^{25}$. Mais il est permis de penser qu'une procédure permettant au juge de prendre directement connaissance des éléments établissant, selon l'exécutif, la nécessité d'une surveillance de masse des réseaux informatiques offrirait un niveau de garantie contre l'arbitraire sensiblement plus important.

11 Mais c'est plus encore la conception extensive que retient le juge administratif de la « sécurité nationale » qui est de nature à émousser la portée de son contrôle.

\section{B. L'extension des motifs de surveillance}

12 C'est essentiellement s'agissant des motifs permettant d'ordonner la conservation généralisée et indifférenciée de nos données de connexion que la mise à l'écart des exigences européennes au profit du cadre constitutionnel aboutit à un relâchement significatif de l'encadrement des services de renseignement, en leur permettant de recourir plus largement à une telle surveillance de masse. On sait en effet que la Cour de justice de l'Union européenne définit les menaces pour la sécurité nationale comme les «activités de nature à déstabiliser gravement les structures constitutionnelles, politiques, économiques ou sociales fondamentales d'un pays, et en particulier à menacer directement la société, la population ou l'État en tant que tel, telles que notamment des activités de terrorisme ", précisant qu'elles doivent être caractérisées par « des circonstances suffisamment concrètes » et, surtout, qu'elles « se distinguent, par leur nature et leur particulière gravité, du risque général de survenance de tensions ou de troubles, même graves, à la sécurité publique ${ }^{26}$. En se référant quant à lui à l'objectif de valeur constitutionnelle de « sauvegarde des intérêts fondamentaux de la Nation ", le Conseil d'État est conduit à autoriser le recours à la surveillance de masse 
pour l'ensemble des motifs prévus à l'article L. 811-3 du code de la sécurité intérieure, lequel vise notamment la prévention "des actions tendant au maintien ou à la reconstitution de groupements dissous", "des violences collectives de nature à porter gravement atteinte à la paix publique» ou, encore, "de la criminalité et de la délinquance organisées » ${ }^{27}$. Soit, précisément, des faits relevant du "risque général de troubles graves à la sécurité publique » qui, selon le juge européen, ne peuvent justifier la conservation générale et indifférenciée de nos données de connexion.

Sans surprise, ce sont principalement à de tels troubles que se réfère le Conseil pour caractériser l'actualité de la menace à la sécurité nationale, relevant « la persistance d'un risque terroriste élevé, ainsi qu'en témoigne notamment le fait que sont survenues sur le sol national au cours de l'année 2020 six attaques abouties ayant causé sept morts et onze blessés", ainsi qu'une "augmentation de l'activité de groupes radicaux et extrémistes (sic) ». En outre, le juge administratif prend en compte des éléments qui tiennent moins à l'existence d'une menace concrète qu'aux structures économiques et administratives de la France, estimant qu'elle apparaît "particulièrement exposée au risque d'espionnage et d'ingérence étrangère, en raison notamment de ses capacités et de ses engagements militaires et de son potentiel technologique et économique ${ }^{28}$. Dans une telle perspective, on peine à voir par quel miracle la surveillance de masse des réseaux informatiques puisse un jour cesser d'être considérée comme justifiée...

C'est également en se référant aux objectifs de valeur constitutionnelle de " prévention des atteintes à l'ordre public » et de « recherche des auteurs d'infractions pénales » que le Conseil d'État est conduit à neutraliser les exigences de la Cour de Luxembourg en ce qu'elle prohibe le recours à la conservation généralisée et indifférenciée des données de connexion pour d'autres motifs que la prévention des atteintes à la sécurité nationale. En une formule sans équivoque, les juges du Palais-Royal affirment ainsi que ces objectifs ne sauraient être atteints sans une telle surveillance, considérant à cet égard que la possibilité d'imposer en la matière une obligation de conservation ciblée qu'autorise la Cour de justice de l'Union européenne - serait insuffisante ${ }^{29}$. Toutefois, par une astuce rhétorique dont il a le secret, le Conseil juge que dans la mesure où la conservation généralisée est par ailleurs justifiée pour les besoins de la sécurité nationale et qu'il est loisible aux autorités judiciaires et administratives de requérir des opérateurs, pour les besoins de leurs missions, l'accès aux données ainsi conservées, «le Gouvernement ne pouvait pas imposer aux opérateurs de communications électroniques, aux fournisseurs d'accès à internet et aux hébergeurs la conservation généralisée et indifférenciée des données de connexion [...] aux fins de lutte contre la criminalité et de prévention des menaces à l'ordre public sans méconnaître le droit de l'Union européenne». Mais s'il donne en apparence des gages au juge européen, c'est pour mieux préciser que cette solution ne vaut que dans la mesure où "l'existence d'une menace grave sur la sécurité nationale justifie la conservation généralisée et indifférenciée des données de connexion ${ }^{30}$. Dans l'hypothèse (d'école) où cette justification disparaitrait, la surveillance de masse pourrait alors être justifiée par la lutte contre la criminalité et les menaces graves à l'ordre public. En privilégiant une lecture souverainiste plutôt que garantiste du cadre constitutionnel, la solution adoptée par le Conseil d'État est ainsi de nature à amoindrir sensiblement le droit à la sûreté des citoyens. 


\section{II - Une conception souverainiste des exigences constitutionnelles}

16 Si elle s'inscrit dans une conception classique et attendue de la hiérarchie des normes en droit français (A), la solution adoptée par le Conseil d'État questionne fortement en ce que, pour la première fois à notre connaissance, une règle constitutionnelle est mobilisée pour faire échec à l'application d'une norme internationale ayant pour objet de renforcer les droits fondamentaux des citoyens (B).

\section{A. Une conception traditionnelle de la hiérarchie des normes}

Selon la formule consacrée, le Conseil d'État considère que « la suprématie conférée par l'article 55 de la Constitution aux engagements internationaux ne s'applique pas, dans l'ordre interne, aux dispositions de nature constitutionnelle $\aleph^{31}$. Une telle interprétation fait logiquement primer les normes constitutionnelles sur les normes internationales ayant vocation à s'appliquer directement en droit interne. Elle est du reste partagée tant par le Conseil constitutionnel ${ }^{32}$ que par la Cour de cassation ${ }^{33}$. C'est pourquoi, dès lors que ce moyen lui était opposé par le gouvernement, c'est sans surprise que le juge administratif a été conduit à considérer que les normes constitutionnelles devaient primer sur les dispositions des traités européens telles qu'interprétées par la Cour de justice de l'Union européenne. On sait que cette dernière juge au contraire - et de longue date - que la primauté du droit de l'Union s'impose à toute norme de droit interne, fût-elle de nature constitutionnelle ${ }^{34}$. Une interprétation qui procède de l'économie générale de la construction européenne, fondée non sur une logique intergouvernementale mais bien sur une logique fédérale, dès lors que la législation commune est adoptée à la majorité. C'est pourquoi la conception française de la hiérarchie des normes ne laisse pas de surprendre nombre de nos voisins, tant elle porte en germe la remise en cause du principe même de l'Union: si l'on admet que la Constitution d'un État membre puisse faire échec à l'application du droit européen, c'est toute la pérennité de l'édifice commun qui est menacée.

Toutefois, on ne saurait balayer d'un revers de la main la problématique comme l'expression d'un souverainisme suranné. En effet, la construction européenne comporte depuis l'origine une dimension proprement technocratique qui fait des institutions un moyen non d'associer, mais de contourner la souveraineté des États membres pour imposer des réformes économiques sans passer par la délibération démocratique. Une dynamique dont le droit communautaire constitue la cheville ouvrière, faisant de la Cour de justice de l'Union européenne l'instrument d'une «hyperconstitutionnalisation » de politiques publiques qui devraient être laissées à l'appréciation des peuples ${ }^{35}$. C'est précisément pour compenser un tel «déficit démocratique » - pour reprendre la bien pudique formule consacrée - que, de longue date, la Cour constitutionnelle allemande veille à la compatibilité des exigences de l'intégration européenne avec la loi fondamentale, proposant une lecture du principe de subsidiarité "sous l'angle des droits démocratiques du, ou plutôt des peuple(s) souverain(s) $\aleph^{36}$. Une approche particulièrement cohérente d'un point de vue démocratique : la délégation consentie à nos représentants n'ayant d'autre objet que la garantie de nos libertés en société ${ }^{37}$, les accords internationaux qu'ils adoptent en notre nom ne sauraient avoir pour effet d'en limiter la portée. Dans une telle perspective, la 
solution adoptée par le Conseil d'État dans son arrêt du 21 avril 2021 est porteuse d'indéniables avancées. Elle signifie en effet qu'aucune norme internationale - qu'il s'agisse du droit de l'Union ou encore d'un accord de "libre-échange " comme le CEUTA - ne peut s'appliquer dans notre ordre juridique si elle menace une liberté constitutionnellement garantie. Que l'on songe seulement aux droits économiques et sociaux que la Constitution protège en tant que "principes particulièrement nécessaires à notre temps ", et l'on mesure le potentiel de déstabilisation de l'ordre juridique commercial que porte la décision du Conseil.

Tel n'est malheureusement pas l'horizon aujourd'hui ouvert par ce dernier. En considérant que les normes constitutionnelles peuvent être mobilisées pour justifier les atteintes à nos libertés, il en adopte au contraire une conception singulièrement répressive.

\section{B. Une conception répressive des exigences constitutionnelles}

Lors de son adoption formelle par l'arrêt Arcelor, c'est pour veiller au respect du principe d'égalité devant la loi que le Conseil d'État s'est autorisé à vérifier si la mise à l'écart d'une norme nationale contraire au droit européen ne remettait pas en cause les exigences constitutionnelles. Dans la présente décision, ce raisonnement est en quelque sorte mis en œuvre à front renversé, pour faire échec à une jurisprudence visant au contraire à renforcer la protection du droit à la vie privée des citoyen-ne-s français-e-s. Alors que l'abondante jurisprudence relative au «dialogue des juges» en matière de droits fondamentaux entamée depuis l'arrêt Solange $1^{38}$ avait, jusqu'à présent, été essentiellement utilisée par les juridictions suprêmes pour renforcer la protection des droits fondamentaux contre le droit de l'Union européenne, c'est à notre connaissance la première décision d'une cour suprême qui se fonde sur une constitution nationale pour limiter un droit fondamental garanti par le droit de l'Union européenne.

Plus largement, alors que la Constitution s'analyse - du moins dans une perspective démocratique - comme un outil permettant de prévenir l'arbitraire des pouvoirs publics, le Conseil d'État en fait ici l'instrument permettant au contraire d'émousser l'encadrement du pouvoir répressif tel qu'il résulte des exigences européennes. Certes, les juges du Palais-Royal prennent le soin de préciser que les objectifs à valeur constitutionnelle au nom desquels il réduit la portée de ces exigences sont "nécessaires à la sauvegarde de droits et de principes de même valeur ${ }^{39}$. Mais ils oublient ce faisant que, dans une société démocratique, les mesures répressives doivent non seulement poursuivre un but légitime, mais encore et surtout être strictement nécessaires et proportionnées au but recherché, qu'il s'agisse de l'élucidation des infractions ou de la prévention des atteintes à la sécurité nationale. En outre, c'est peu dire qu'après trente ans d'inflation normative, notre système répressif poursuit bien d'autres fins que la seule prévention et la sanction des atteintes aux libertés des citoyen-ne-s ${ }^{40}$. En définitive ce n'est que dans l'hypothèse où une norme internationale ferait en soi obstacle à toute possibilité de prévenir ou de sanctionner une atteinte avérée à nos droits et libertés que l'on pourrait considérer qu'elle met en péril notre droit à la sûreté. À l'évidence, tel n'est pas le cas de la jurisprudence de la Cour de justice de l'Union européenne en matière de conservation des données de connexion informatique... 
La solution adoptée par le Conseil d'État n'est pas davantage assimilable à la jurisprudence de la Cour européenne des droits de l'homme en matière de droit à la vie. Si cette dernière a pu considérer que ce droit était de nature à justifier des atteintes à la liberté individuelle particulièrement importantes et même préventives ${ }^{41}$, elle ne s'est jamais prononcée que dans des cas d'espèce très particuliers. Au contraire, en se référant à de simples objectifs à valeur constitutionnelle - qui ne consacrent en tant que tels aucun droit, mais se bornent à assigner des finalités au législateur - le Conseil d'État consacre une conception de la Constitution qui en fait un outil générique de limitation de nos libertés. À l'heure où certains gouvernements européens, comme celui de la Pologne, envisagent très sérieusement de se servir de leur loi fondamentale pour s'opposer à l'invalidation, par la Cour de justice de l'Union européenne ${ }^{42}$, des réformes remettant en cause l'indépendance de la Justice, on ne saurait trop souhaiter que le juge administratif fasse évoluer sa propre jurisprudence vers une protection accrue des droits fondamentaux.

Les Lettres « Actualités Droits-Libertés » (ADL) du CREDOF (pour s'y abonner) sont accessibles sur le site de la Revue des Droits de l'Homme (RevDH) - Contact

\section{NOTES}

1. Voir Théodore Christakis, "French Council of state discovers the "philosopher stone " of data retention ", About:intel, 23 avril 2021, https://aboutintel.eu/france-council-of-state-ruling/

2. Shahin Vallée, Gerard Genevoix, « A Securitarian Solange: France has launched a cluster bomb on the EU's legal and political order " VerfBlog, 2021/4/25, https://verfassungsblog.de/asecuritarian-solange/

3. Jacques Ziller, «The Conseil d'Etat refuses to follow the Pied Piper of Karlsruhe » VerfBlog, 2021/4/24, https://verfassungsblog.de/the-conseil-detat-refuses-to-follow-the-pied-piper-ofkarlsruhe

4. CJUE [GC], 8 avril 2014, Digital Rights Ireland Ltd \& Michael Seitlinger e.a., aff j. C-293/12 \& C-594/12. V. Florence Benoit-Rohmer, « Protection des données personnelles», RTDE 2015, p.168; Denys Simon, «La révolution numérique du juge de l'Union: les premiers pas de la cybercitoyenneté », Europe n ${ }^{\circ}$ 7, p. 4 ; Marie-Laure Basilien-Gainche, « Une prohibition européenne claire de la surveillance électronique de masse », in Revue des droits de l'homme, 14 mai 2014.

5. UK High Court, David Davis and others -v- Secretary of State for the Home Department, 17 juillet 2015

6. CJUE, Gr. Ch., 6 octobre 2015, Maximilian Schrems c. Data Protection Commissioner, aff. C362/14 ;

7. CJUE, 21 décembre 2016, Tele2 Sverige et Watson (C203/15 et C698/15) ; CJUE (grande chambre), 6 octobre 2020, Quadrature du Net et autres, C511/18, C512/18 et C520/18. 
8. Voir sur cet arrêt Vincent Sizaire, «Savoir resserrer les mailles du filet ", La Revue des droits de l'homme [En ligne], Actualités Droits-Libertés, Publié le 28 novembre 2020. URL: http:// journals.openedition.org/revdh/10627; DOI: https://doi.org/10.4000/revdh.10627

9. Voir Jean-Baptiste Jacquin, «Paris pousse le Conseil d'État à défier la justice européenne sur les données de connexion, ", Le Monde, 8 avril 2021

10. Soit les articles L.851-1 et suivants du code de la sécurité intérieure.

11. Dans une décision du 22 avril 2021, la Cour Constitutionnelle a procédé à une lecture radicalement différente de la décision de la CJUE, et annulé la législation nationale sur la conservation des données de connexion. Voir Cour Constitutionnelle de Belgique, Arrêt n57-2021 du 22 avril 2021,

12. C'est-à-dire les données d'identité, qui permettent d'identifier l'utilisateur d'un moyen de communication électronique (par exemple les nom et prénom liés à un numéro de téléphone ou l'adresse IP par laquelle un utilisateur se connecte à internet), les données relatives au trafic, qui permettent de retracer les dates, heures et destinataires des communications électroniques, ou la liste des sites internet consultés et les données de localisation, qui recensent les antennes relais auxquelles ont été connectés les appareils.

13. CE, Assemblée, 21 avril 2021, FRENCH DATA NETWORK et autres, 393099, n8.

14. CE, Assemblée, 8 février 2007, Société Arcelor Atlantique Lorraine et autres, $n^{\circ} 287110$, p. 55

15. Ibid., $\mathrm{n}^{\circ} 6$ et 7 .

16. Ibid., $\mathrm{n}^{\circ} 9$ et 10 .

17. Ibid., $\mathrm{n}^{\circ} 74,77,80$ et 83 .

18. CJUE, Gr.Ch., 6 octobre 2015, Maximilian Schrems c. Data Protection Commissioner, Aff. C362/14

19. CE, Assemblée, 21 avril 2021, FRENCH DATA NETWORK et autres, 393099, $\mathrm{n}^{\circ} 91$.

20. Cour EDH, 24 avril 1990, Huvig contre France, Req. $\mathrm{N}^{\circ} 11105 / 84$, § 34 ; Cour EDH, 16 février 2000, Amann c. Suisse, Req. $\mathrm{N}^{\circ}$ 27798/95, §§ 56-58; Cour EDH, 22 juin 2006, Weber et Saravia c. Allemagne, Req. 54934/00, § 95.

21. CE, Assemblée, 21 avril 2021, FRENCH DATA NETWORK et autres, 393099, n4 45.

22. Article L. 773-1 du Code de Justice Administrative.

23. CE, 20 février 2012, MINISTRE DE LA DEFENSE ET DES ANCIENS COMBATTANTS, 350382, A.

24. CE 29 mai 1970, Min. Finances c/ Nguyen-Quang, Lebon p.377.

25. CE, 21 nov. 2007, Mme Tharaud, req. No 258820.

26. CJUE (grande chambre), 6 octobre 2020, Quadrature du Net et autres, C511/18, C512/18 et C520/18, § 135 à 137 et 147

27. CE, Assemblée, 21 avril 2021, FRENCH DATA NETWORK et autres, 393099, n¹9.

28. Ibid., $\mathrm{n}^{\circ} 44$.

29. Ibid., $\mathrm{n}^{\circ} 57$.

30. Ibid., $\mathrm{n}^{\circ} 58$.

31. CE, Sarran et Levacher et autres, 30/10/1998, 200286(...)

32. CC, décision n92-315 DC, 12 janvier 1993, cons. 4 et 5, Journal officiel du 14 janvier 1993, page 777, Rec. p. 9.

33. Cass., Assemblée plénière, 2 juin 2000, pourvoi $n^{\circ} 99-60.274$, Bulletin A.P. $1999, n^{\circ} 4$.

34. CJCE, 17 décembre 1970, Internationale Handelsgesellschaft; CJCE, 9 mars 1978, Simmenthal.

35. Dieter Grimm, «Quand le juge dissout l'électeur », Le monde diplomatique, juillet 2017.

36. Isabelle Bourgeois, " La Cour de Karlsruhe, un think tank pour l'Europe », Revue internationale et stratégique, vol. 90, $\mathrm{n}^{\circ} 2,2013$, pp. 28-38.

37. Article 2 de la Déclaration des droits de l'homme et du citoyen du 26 août 1789.

38. Voir Édouard Dubout et Sébastien Touzé (dir.), Les droits fondamentaux : charnières entre ordres et systèmes juridiques, Paris, Pedone, 2010.

39. CE, Assemblée, 21 avril 2021, FRENCH DATA NETWORK et autres, 393099, n9.

40. Vincent Sizaire, Sortir de l'imposture sécuritaire, La Dispute, Paris, 2016. 
41. Raphaële Parizot, «Prévention du meurtre : la Cour européenne des droits de l'homme va-telle trop loin?», D. 2013. 188.

42. "La Cour de justice de l'UE et la Pologne croisent le fer sur l'indépendance des magistrats », Le Monde, 7 mai 2021.

\section{ABSTRACTS}

Appelé à appliquer les décisions de la Cour de justice de l'Union européenne ayant sévèrement restreint les possibilités de surveillance de masse de la population, le Conseil d'État choisit de ne prendre en compte les exigences européennes que dans la mesure où elles restent compatibles avec le cadre constitutionnel français. Si cette approche le conduit en l'espèce à reprendre à son compte une large part de la jurisprudence du juge de Luxembourg, censurant en conséquence le cadre juridique national régissant la conservation des données de connexion des internautes, elle véhicule une forme de souverainisme juridique qui fragilise la protection européenne des droits et libertés des citoyen-ne-s.

\section{AUTHORS}

\section{VINCENT SIZAIRE}

Maître de conférences associé à l'Université Paris Nanterre

JEAN-PHILIPPE FOEGLE

Doctorant en droit public au Centre de Théorie et d'Analyse du Droit, équipe CREDOF 